\title{
A NOTE ON LINEAR TOPOLOGICAL SPACES*
}

\author{
D. H. HYERS
}

A space $T$ is called a linear topological space if (1) $T$ forms a linear $\dagger$ space under operations $x+y$ and $\alpha x$, where $x, y \in T$ and $\alpha$ is a real number, (2) $T$ is a Hausdorff topological space, $\ddagger(3)$ the fundamental operations $x+y$ and $\alpha x$ are continuous with respect to the Hausdorff topology. The study $\S$ of such spaces was begun by A. Kolmogoroff (cf. [4]. Kolmogoroff's definition of a linear topological space is equivalent to that just given). Kolmogoroff calls a set $S \subset T$ bounded, if for any sequence $x_{\nu} \epsilon S$ and any real sequence $\alpha_{\nu}$ converging to 0 we have $\lim _{\nu \rightarrow \infty} \alpha_{\nu} x_{\nu}=\theta$, where $\theta$ is the zero element of $T$. He then shows that a linear topological space $T$ reduces to a linear normed space $\|$ if and only if there exists in $T$ an open set which is both convex $\mathbb{T}$ and bounded. In this note, the characterization of other types of spaces among the class of linear topological spaces is studied. Spaces which are locally bounded, that is spaces containing a bounded open set, are found to be "pseudo-normed" on the one hand, and metrizable on the other, but not in general normed. Fréchet spaces, or spaces of type $(\mathrm{F})$, are characterized. The main result of the paper is that a linear topological space $T$ is finite dimensional, and hence linearly homeomorphic to a finite dimensional euclidean space, if and only if $T$ contains a compact, open set. This of course is a generalization to linear topological spaces of the well known theorem of $\mathrm{F}$. Riesz for the space of continuous functions.

We first give some needed properties of bounded sets. The following notations will be used throughout. We denote by $\alpha S$ the set of all $\alpha x$ with $x \in S$; by $x+S$, the set of all elements $x+y$ where $y$ ranges over $S$; by $S_{1}+S_{2}$, the set of all $x+y$ with $x \in S_{1}, y \epsilon S_{2}$.

THEOREM 1. A set $S$ of a linear topological space is bounded if and only if, given any neighborhood $U$ of the origin, there is an integer $\nu$ such that $|\alpha|<1 / \nu$ implies $\alpha S \subset U$.

* Presented to the Society, April 3, 1937.

† Cf., for example, [1], p. 26. Numbers in brackets refer to the bibliography at the end of the paper.

$\ddagger$ Cf. [2], pp. 228-229, axioms (A), (B), (C), (5); or [3], pp. 43 and 67.

$\S$ Important instances of a linear topological space were studied by J. von Neumann several years before Kolmogoroff's paper was published. Cf. Mathematische Annalen, vol. 102 (1930), pp. 370-427. See also [5].

\| Cf. [1], p. 53.

TI A set $S$ is convex if and only if $x, y \in S$ and $0<\alpha<1$ imply $\alpha x+(1-\alpha) y \epsilon S$. 
Proof. The condition of the theorem is sufficient, for suppose that it is satisfied. Then if $\alpha_{\lambda} \rightarrow 0, x_{\lambda} \epsilon S$, we see, for any chosen neighborhood $U$ of the origin $\theta$, that $\alpha_{\lambda} x_{\lambda} \in U$ for all $\lambda$ for which $\left|\alpha_{\lambda}\right|<1 / \nu$. That is, $\lim _{\lambda \rightarrow \infty} \alpha_{\lambda} x_{\lambda}=\theta$. The condition is necessary. For, assume that $S$ is bounded, but that the condition of the theorem does not hold. Then for some $U$ and any integer $\nu>0$, there exists $x_{\nu} \in S$, and a real $\alpha_{\nu}$ with $\left|\alpha_{\nu}\right|<1 / \nu$ such that $\alpha_{\nu} x_{\nu} \bar{\epsilon} U$. Therefore $\alpha_{\nu} \rightarrow 0$ with $1 / \nu$, but $\alpha_{\nu} x_{\nu}$ does not converge to $\theta$. That is, $S$ is not bounded. This contradiction proves the necessity of the condition.

This theorem provides us with an alternative definition of boundedness. For other equivalent definitions see [5] and [6]. See also [7].

Theorem 2. A compact set of a linear topological space is bounded.

Proof. Let $S \subset T$ be compact, so that every infinite subset of $S$ has a limit point in $T$. Assume, contrary to the theorem, that $S$ is not bounded. Let $U$ be any chosen neighborhood of the origin $\theta$. By denying the definition of boundedness, we are led to a sequence $\alpha_{\nu} \rightarrow 0$ and a sequence $x_{\nu} \epsilon S$, with $\alpha_{\nu} x_{\nu} \bar{\epsilon} U$, for $\nu=1,2, \cdots$. But, since $S$ is compact, there is a limit point $p$ of the infinite sequence $x_{\nu}$. By the continuity of the function $\alpha x$ at $\alpha=0, x=p$, there exists a $\delta>0$ and a neighborhood $V$ of $p$ such that $\alpha V \subset U$ for $|\alpha|<\delta$. Since $p$ is a limit point of the sequence $x_{\nu}$, there is an infinite subsequence $x_{\nu_{\kappa}}$ such that $x_{\nu_{\kappa}} \in V$ for all $\kappa$. For sufficiently large $\kappa$ we have $\left|\alpha_{\nu_{\kappa}}\right|<\delta$, and therefore $\alpha_{\nu_{\kappa}} x_{\nu_{\kappa}} \epsilon \alpha_{\nu_{\kappa}} V \subset U$, contrary to the fact that $\alpha_{\nu} x_{\nu} \bar{\epsilon} U$. This contradiction proves the theorem.

Definition. A linear space $E$ will be said to be pseudo-normed if corresponding to each $x \in E$ there is a real number $|x|$ with the properties :

(i) $|x| \geqq 0 ;|x|=0$ implies $x=\theta$,

(ii) $|\alpha x|=|\alpha||x|$ (where $|\alpha|$ is the absolute value of $\alpha$ ),

(iii) if $|x| \rightarrow 0,|y| \rightarrow 0$, then $|x+y| \rightarrow 0$.

A linear topological space $T$ will be called pseudo-normable if it can be pseudo-normed in such a way that the topology according to the pseudo-norm is equivalent to the original Hausdorff topology.

THEOREM 3. Every pseudo-normed linear space is a locally bounded linear topological space. Conversely, if a linear topological space contains a bounded open set, then the space is pseudo-normable.

Proof: Let $E$ be a pseudo-normed space and for any chosen $\alpha$ consider the "sphere" $|y-x|<\alpha$. Denote by $U(x ; \alpha)$ the set of points $z$ of this sphere for each of which there exists a positive $\delta$ such that 
$|w-z|<\delta$ implies $|w-x|<\alpha$. It is easy to verify that $E$ is a Hausdorff space with neighborhoods $U(x ; \alpha)$, and that the operations of vector addition and scalar multiplication are continuous, so that $E$ is a linear topological space. Property (iii) of the pseudo-norm is used in proving both the Hausdorff separation axiom and the continuity of addition. Local boundedness now follows from property (ii).

On the other hand, let $T$ be a locally bounded linear topological space, and suppose that $U$ is a bounded open set. Without loss of generality we may suppose that $U$ contains the origin, and that $U=(-1) U$. Then the sets $\alpha U, \alpha \neq 0$, are all open, and clearly form a complete neighborhood system of the origin. Put $|x|=$ g.l.b. $|\alpha|$, $x \in \alpha U$, and it follows without difficulty that the postulates for a pseudo-normed space are satisfied. Next, for any integer $\nu>0$ consider the set $|x|<1 / \nu$. By definition of $|x|$, this is the set of all $x$ such that $x \epsilon \alpha U,|\alpha|<1 / \nu$, and hence* the set $|x|<1 / \nu$ is open. Now by Theorem 1 and the fact that $U$ is bounded, for any neighborhood $V$ of the origin there is an integer $\nu=\nu(V)$ such that $|\alpha|<1 / \nu$ implies $\alpha U \subset V$. Hence the sphere $|x|<1 / \nu$, being the union of the sets $\alpha U$ for which $|\alpha|<1 / \nu$, is contained in $V$, and the spheres $|x|<1 / \nu$ form a complete neighborhood system of the origin. Thus the topology according to the pseudo-norm is equivalent to the original Hausdorff topology.

Corollary 1. A locally bounded linear topological space satisfies the first countability axiom.

CoROllary 2. If $T$ is locally bounded, then a set $S \subset T$ is bounded if and only if there is a $\mu>0$ such that $x \in S$ implies $|x|<\mu$.

TheOREM 4. A necessary and sufficient condition that a linear topological space be finite dimensional is that it contain a non-empty set which is both open and compact.

Proof. The necessity is immediate, since every finite dimensional linear topological space (a linear space with a "finite basis" $\left(x_{1}, \cdots, x_{\nu}\right)$ such that every element is uniquely expressible in the form $\left.\sum_{\lambda=1}^{\nu} \alpha_{\lambda} x_{\lambda}\right)$ is linearly homeomorphic to a finite dimensional euclidean space. $\dagger$ To prove the sufficiency, let $U$ be a compact open set containing the origin. By Theorem 2,U is bounded, and hence $T$ is pseudo-normable by Theorem 3. If $S$ is any bounded set of $T$,

* [7], Theorem 1.2. Special use is made of the continuity of $\alpha x$ in proving this theorem.

$\dagger$ For the proof see [8]. 
then for some $\alpha$ we have $S \subset \alpha U$ (Theorem 1), so that $S$ is compact, being a subset of the compact set $\alpha U$. Also, from Corollary 1 , every limit point is the limit of a convergent sequence. Hence every bounded sequence has a convergent subsequence, where the convergence is taken according to the pseudo-norm. From here on, with the aid of Corollary 2, the proof follows that of a similar result for the linear normed space of continuous functions due to F. Riesz, ${ }^{*}$ and will be omitted.

CoRollary 3. A linear topological space $T$ is linearly homeomorphic to a finite dimensional euclidean space if and only if $T$ is locally compact.

A theorem on metrizability of "Hausdorff groups" has been proved by G. Birkhoff (see [9]), which states that a Hausdorff group is metrizable if and only if the first countability axiom is satisfied. Since a linear topological space is a commutative Hausdorff group under addition, we are led to the following theorem, characterizing spaces of type $(F)$. For the definition of these spaces see [1], page 35 .

Theorem 5. A space $E$ is a space of type $(F)$ if and only if $E$ is a sequentially complete $\dagger$ linear topological space which satisfies the first countability axiom.

Proof. Let $T$ be a "sequentially complete" linear topological space, satisfying the first countability axiom. Then by G. Birkhoff's metrization theorem for Hausdorff groups, $T$ is metrizable. Moreover the metric $(x, y)$ exhibited by Birkhoff can be easily shown to have the property $(x-y, \theta)=(x, y)$. Therefore $T$ is a linear space with a metric $(x, y)$ satisfying $(x-y, \theta)=(x, y)$ and such that $\alpha x$ is continuous on both the left and the right. Hence, since $T$ is supposed complete, it is a space of type $(F)$.

Conversely, let $E$ be a space of type $(F)$, so that $E$ is a complete metric space with a metric $(x, y)$ which is invariant under translation, and such that the function $\alpha x$ is continuous in $\alpha$ and $x$ separately. In order to prove that $E$ is a linear topological space, it is sufficient to show that $\alpha x$ is a continuous function of its two variables jointly. This may be established by an argument involving sets of the second category, very much like that used by D. Montgomery $\ddagger$ in a similar connection, and the details will be omitted here.

From Corollary 1 we have the following corollary:

* [11], p. 75, Lemma 2, and p. 78, Lemma 5.

$\dagger[5]$, p. 10.

$\ddagger[10]$. See especially the proof of the lemma on p. 880 . 
Corollary 4. A locally bounded linear topological space is metrizable by means of a metric satisfying $(x, y)=(x-y, \theta)$.

From Theorem 3 and Corollary 4 we have the result stated in the introduction, namely, that a locally bounded linear topological space is both pseudo-normable and metrizable, the topologies according to the pseudo-norm, the metric, and the original Hausdorff neighborhoods being equivalent. However, not every locally bounded space is normable, as is shown by the pseudo-normed space $H_{1 / 2}$ of all infinite dimensional vectors $x=\left(x_{1}, x_{2}, x_{3}, \ldots\right)$ for which the series $\sum_{\nu=1}^{\infty}\left|x_{\nu}\right|^{1 / 2}$ converges, and where $|x|=\left\{\sum_{\nu=1}^{\infty}\left|x_{\nu}\right|^{1 / 2}\right\}^{2}$. For Tychonoff has shown* that this space is not locally convex, and hence not normable, by Kolmogoroff's normability theorem.

\section{REFERENCES}

1. S. Banach, Opérations Linéaires, Warsaw, 1932.

2. F. Hausdorf, Mengenlehre, Berlin, 1927.

3. Alexandroff and Hopf, Topologie, vol. 1, Berlin, 1935.

4. A. Kolmogoroff, Zur Normierbarkeit eines topologischen linearen Raumes, Studia Mathematica, vol. 5 (1934), pp. 29-33.

5. J. von Neumann, On complete topological spaces, Transactions of this Society, vol. 37 (1935), pp. 1-20.

6. A. D. Michal and E. W. Paxson, The differential in abstract linear spaces with a topology, Comptes Rendus of the Warsaw Academy of Sciences (in press; for a resumé see Comptes Rendus of the Paris Academy, vol. 202, pp. 1741-1743).

7. D. H. Hyers, California Institute of Technology Thesis, 1937 (at California Institute of Technology Library).

8. A. Tychonoff, Ein Fixpunktsatz, Mathematische Annalen, vol. 111 (1935), pp. 767-776.

9. G. Birkhoff, A note on topological groups, Compositio Mathematica, vol. 3 (1936), pp. $427-430$.

10. D. Montgomery, Continuity in topological groups, this Bulletin, vol. 42 (1936), pp. $879-882$.

11. F. Riesz, Über lineare Funktionalgleichungen, Acta Mathematica, vol. 41 (1918), pp. 71-98.

UNIVERSITY OF WisCONSIN

* [8], pp. 768-769. 